\title{
Assessment of Cardiovascular Risk in People Living with Epilepsy
}

\author{
Kotha Lakshmi Sravanthi ${ }^{1}$, Gopal Krushna Pal ${ }^{1}{ }^{1}$, Pradeep Pankajakshan Nair ${ }^{2}$, Rajasegaran Rajalakshmi ${ }^{1}$, \\ Nivedita Nanda ${ }^{3}$
}

\section{Kotha Lakshmi Sravanthi', Gopal Krushna Pal ${ }^{1, *}$, Pradeep Pankajakshan Nair ${ }^{2}$, Rajasegaran Rajalakshmi' ${ }^{1}$, Nivedita $\mathrm{Nanda}^{3}$}

'Department of Physiology, Jawaharlal Institute of Postgraduate Medical Education and Research (JIPMER), Puducherry, INDIA.

${ }^{2}$ Department of Neurology and Jawaharlal Institute of Postgraduate Medical Education and Research (JIPMER), Puducherry, INDIA. ${ }^{3}$ Department of Biochemistry, Jawaharlal Institute of Postgraduate Medical Education and Research (JIPMER), Puducherry, INDIA.

\section{*Correspondence}

\section{Dr. Gopal Krushna Pal}

Senior Professor and Program

Director, Advance Center of Yoga,

Department of Physiology, Jawaharlal Institute of Postgraduate Medical Education and Research (JIPMER), Puducherry-605 006, INDIA.

Phone: +91- 9344291160

Email: drgkpal@gmail.com

History

- Submission Date: 15-07-2021;

- Review completed: 05-08-2021;

- Accepted Date: 27-08-2021.

DOI : 10.5530/ijcep.2021.8.3.28

Article Available online

http://www.ijcep.org/v8/i3

\section{Copyright}

(C) 2021 Phcog.Net. This is an openaccess article distributed under the terms of the Creative Commons Attribution 4.0 International license.

\begin{abstract}
Background and Aim: Increase prevalence of cardiovascular morbidity and mortality has been reported in people living with epilepsy (PWE) compared to the general population. This study was done to assess the cardiovascular risk of PWE using Heart rate variability (HRV) and Blood Pressure variability (BPV) techniques. Methods: Twenty-seven patients with epilepsy and thirty age and gender-matched healthy individuals were included in the study and control group, respectively. Basal cardiovascular parameters (heart rate-HR, systolic blood pressureSBP, diastolic blood pressure-DBP), HRV indices, and BPV parameters were recorded and compared between the study and control group individuals. In addition, the association of the Total Power (TP) of HRV with other study parameters was assessed by Pearson correlation coefficient test. Results: Cardiovascular parameters such as HR, SBP, DBP, rate pressure product, cardiac output and total peripheral resistance were significantly high in PWE compared to healthy individuals. Baroreflex sensitivity (BRS) and HRV parameters indicative of cardiac parasympathetic drive (TP, SDNN, RMSSD, and pNN50) were significantly low in these individuals. While a significant negative correlation was observed between TP, BHR and DBP, a significant positive correlation was observed between TP and BRS. Conclusion: Cardiac sympathovagal imbalance characterized by increased sympathetic and decreased parasympathetic activity was evident in PWE. These changes in the autonomic nervous system could predispose them to increased risk of cardiovascular morbidity.

Key words: Epilepsy, Heart rate variability, Cardiovascular risks, People living with epilepsy, Total power of HRV.
\end{abstract}

\section{INTRODUCTION}

Epilepsy is a "disorder characterized by recurrent seizures due to abnormal electrical discharges in the brain". According to International League Against Epilepsy (ILAE), Epilepsy is defined as: 1) At least two unprovoked seizures occurring twenty four hours apart, 2) One unprovoked seizure and probability of further seizures similar to general recurrence risk (at least 60\%) after two unprovoked seizures occurring over the next 10 years, 3) Diagnosis of an epilepsy syndrome. ${ }^{[1]}$

Epilepsy is the most prevalent one among various chronic neurological diseases. Worldwide, over 70 million people are known to suffer from epilepsy, and hence this disorder has been considered a global health priority. ${ }^{[2]}$ In India, the prevalence of epilepsy is about $1 \%$ of the population. Higher prevalence of cardiovascular (CV) risk factors and increased incidence of unanticipated demise have been reported in people living with epilepsy (PWE) in contrast to healthy individuals. Sudden unexpected death is associated with the autonomic imbalance and low cardiovascular fitness. ${ }^{[3]}$

Though there are many reports of cardiovascular morbidities and mortalities in epilepsy, these is paucity of report on heart rate variability (HRV)

analysis in epilepsy. Recently heart rate variability has been used as an assessment tool for CV risks in various clinical disorders. ${ }^{[4]}$ Therefore, in the present study, we have performed HRV analysis for $\mathrm{CV}$ risk assessment in PWE. Total power (TP) of HRV has been documented as an important marker of cardiovagal modulation, and decreased TP is an established CV risk. ${ }^{[4]}$ Rate-pressure product (RPP), the physiological indicator of myocardial oxygen demand and stress, is a sensitive measure of $\mathrm{CV}$ stress. ${ }^{[5]}$ Blood pressure variability (BPV) analysis with the estimation of baroreflex sensitivity (BRS) has been used to assess cardiovascular health in many metabolic and neurological disorders. ${ }^{[6]}$ However, till date, there is no report-on BRS of PWE. Therefore, in the present study, we have analyzed the correlation of TP of HRV with RPP and other cardiovascular risk markers.

\section{MATERIALS AND METHODS}

Following approval of the Institute Ethics Committee, 27 epilepsy patients were recruited from the Neurology Outpatient Department of Jawaharlal Institute of Postgraduate Medical Education and Research (JIPMER), Puducherry. Patients

Cite this article: Sravanthi KL, Pal GK, Nair PP, Rajalakshmi R, Nanda N. Assessment of Cardiovascular Risk in People Living with Epilepsy. Int J Clin Exp Physiol. 2021;8(3):118-21. 
diagnosed with epilepsy of less than one-year duration based on the electroencephalogram (EEG) were recruited in the study group. Age and gender-matched healthy subjects were recruited in the control group.

\section{Inclusion and Exclusion Criteria}

Patients aged between 18-60 years, both genders receiving similar group of drugs were included as the study group. Age and gender-matched normal population were recruited as the control group. Participants with comorbidities, pregnancy and mental illness were excluded from the study.

\section{Recording of BPV by Finapres}

After 10 min of supine rest, BRS and other CV parameters such as basal heart rate, systolic blood pressure, diastolic blood pressure, mean arterial pressure, stroke volume, cardiac output, and total peripheral resistance were measured by continuous blood pressure variability method using Finapres (Finometer version 1.22a; Finapres Medical Systems BV, Amsterdam, the Netherlands) based on the principle of Penaz and Wesseling. ${ }^{[7]}$ Rate-Pressure Product (RPP), a determinant of myocardial oxygen consumption and the workload, was calculated using the formula, $\mathrm{RPP}=\mathrm{SBP} \times$ Heart rate $\times 10^{-2} .^{[5]}$

\section{Recording of HRV}

For the recording of short-term HRV, the recommendation of the Task Force on HRV was followed. ${ }^{[4]}$ All experiments were performed in the cardiac autonomic function research laboratory. Short-term HRV was recorded after giving $10 \mathrm{~min}$ of supine rest to the participants. The participants were asked to refrain from heavy physical activity for $24 \mathrm{hr}$ and from consuming alcohol and caffeinated beverages for $12 \mathrm{hr}$ before the recordings. On the day of the test, they were asked to have a light breakfast. The lab temperature was maintained at $25^{\circ} \mathrm{C}-28^{\circ} \mathrm{C}$, and lights were subdued. The participants were asked to void urine before recording and made to lie down comfortably to accustom to the new environment. Initially, heart rate and auscultatory blood pressure were measured after $10 \mathrm{~min}$ of supine rest. Then at supine rest with eyes closed and relaxed position, lead II ECG was acquired at the rate of 200 samples/ second using the BIOPAC MP150 system. Then the data was transferred from BIOPAC to a windows-based PC that had Acqknowledge software version 4.1. Ectopics and artefacts were removed from the ECG recording. With the help of the $\mathrm{R}$ wave detector in the AcqKnowledge software and the Kubios HRV analysis software, extraction of the RR tachogram was done from the $360 \mathrm{sec}$ of edited ECG segment. The RR intervals for the entire recording were taken in the journal and saved in text format. The RR interval data was analysed using the Kubios HRV analysis software version. The software was based on Matlab, and it was compiled to a standalone $\mathrm{C}$ programme using Matlab. A three hundred and sixty seconds data of RR tachogram was used for HRV analysis. The $\mathrm{RR}$ tachogram was interpolated at $2 \mathrm{~Hz}$, and Fast Fourier Transformation (FFT) was computed using Welch periodogram. ${ }^{[8]}$

\section{Statistical Analysis of Data}

SPSS version 22 was used for statistical analysis. All the data were presented as mean $\pm \mathrm{SD}$. Comparison of the continuous data between the two groups (control and study groups) was done using Independent $t$ test. Pearson correlation was done to assess the correlation of TP with various study parameters. All statistical analysis was carried out at a $5 \%$ level of significance and $\mathrm{P}$ value $<0.05$ was considered significant.

\section{RESULTS}

There was no significant difference in the age and anthropometric parameters of the control and the study groups participants, as shown in Cardiovascular parameters like basal heart rate (BHR), systolic blood pressure (SBP), diastolic blood pressure (DBP) and rate pressure product (RPP) were significantly high in the study group compared to the control group, as shown in Table 1.

Table 2 shows the frequency and time domain indices of heart rate variability. Frequency domain index, namely the total power (TP), and time domain indices, namely SDNN, RMSSD, and pNN50, were significantly low in the study group compared to the control group.

Table 3 shows the blood pressure variability parameters. There was a significant increase in cardiac output $(\mathrm{CO})$ and total peripheral resistance (TPR) in the study group compared to the control group.

Table 1: Comparison of anthropometric indices, basal heart rate (BHR), blood pressure (BP), and rate pressure product (RPP) between healthy individuals, control group ( $n=30)$ and patients with epilepsy, study group ( $n=27)$.

\begin{tabular}{cccc}
\hline Variables & Control Group & Study Group & P value \\
\hline Age (years) & $34.78 \pm 3.85$ & $35.15 \pm 4.61$ & 0.300 \\
BMI $\left(\mathrm{Kg} / \mathrm{m}^{2}\right)$ & $24.57 \pm 2.68$ & $25.15 \pm 2.12$ & 0.232 \\
BHR (beats per min) & $71.65 \pm 7.60$ & $78.81 \pm 11.26$ & 0.012 \\
SBP (mm Hg) & $108.30 \pm 5.72$ & $115.85 \pm 8.66$ & 0.015 \\
DBP (mm Hg) & $71.08 \pm 6.30$ & $74.68 \pm 9.66$ & 0.021 \\
MAP (mm Hg) & $83.46 \pm 7.20$ & $87.35 \pm 8.35$ & 0.432 \\
RPP (mm Hg/min) & $77.64 \pm 8.41$ & $91.59 \pm 9.41$ & 0.045 \\
\hline
\end{tabular}

Data expressed as Mean \pm SD.

Comparison between the groups was done by Independent $t$ test

$\mathrm{P}<0.05$ was considered as statistically significant.

BMI: Body Mass Index; BHR: Basal Heart Rate; SBP: Systolic Blood Pressure; DBP: Diastolic Blood Pressure; MAP: Mean Arterial Pressure; RPP: Rate Pressure Product

Table 2: Comparison of heart rate variability (HRV) parameters between healthy individuals, control group $(n=30)$ and patients with epilepsy, study group $(\mathrm{n}=27)$.

\begin{tabular}{cccc}
\hline Variables & Control Group & Study Group & P value \\
\hline FDI of HRV & & & \\
TP $\left(\mathrm{ms}^{2}\right)$ & $1065.70 \pm 305.20$ & $739.52 \pm 122.81$ & $<0.001$ \\
LFnu & $48.10 \pm 15.25$ & $57.71 \pm 16.11$ & 0.77 \\
HFnu & $51.90 \pm 16.78$ & $42.62 \pm 14.05$ & 0.36 \\
LF/HF & $0.92 \pm 0.31$ & $1.37 \pm 0.38$ & 0.28 \\
TDI of HRV & & & \\
SDNN (ms) & $47.20 \pm 14.30$ & $31.00 \pm 7.42$ & 0.001 \\
RMSSD (ms) & $35.41 \pm 10.40$ & $24.93 \pm 5.36$ & 0.001 \\
NN50 & $20.32 \pm 5.36$ & $12.04 \pm 3.88$ & 0.09 \\
pNN50 & $12.20 \pm 2.759$ & $4.94 \pm 0.89$ & $<0.001$ \\
\hline
\end{tabular}

Data expressed as Mean \pm SD.

Comparison between the groups was done by independent $t$ test.

$\mathrm{P}<0.05$ was considered as statistically significant.

TP: Total Power; LFnu: Low frequency component expressed as normalized unit; HFnu: High frequency component expressed as normalized unit; LF:HF- Ratio of low frequency power to high frequency power of heart rate variability; SDNN: Standard Deviation of Normal to Normal interval; RMSSD: Root Mean Square of the Successive Differences of normal to normal interval; NN50: the number of interval differences of successive NN intervals greater than 50ms; pNN50: the proportion derived by dividing NN50 by the total number of NN intervals. 
Table 3: Comparison of blood pressure variability (BPV) parameters between healthy individuals, control group $(n=30)$ and patients with epilepsy, study group ( $n=27)$.

\begin{tabular}{cccc}
\hline Variables & Control Group & Study Group & P value \\
\hline SV $(\mathrm{mL})$ & $72.80 \pm 10.32$ & $81.16 \pm 8.41$ & 0.29 \\
LVET $(\mathrm{ms})$ & $317.50 \pm 20.38$ & $325.97 \pm 23.55$ & 0.44 \\
CO (L/min) & $5.14 \pm 0.58$ & $5.72 \pm 0.88$ & 0.03 \\
TPR (mmHg.min/l) & $1.17 \pm 0.21$ & $1.67 \pm 0.35$ & 0.008 \\
BRS (ms/mmHg) & $21.96 \pm 2.15$ & $14.00 \pm 2.33$ & 0.047 \\
\hline
\end{tabular}

Data expressed as Mean \pm SD.

Comparison between the groups was done by independent $t$ test.

$\mathrm{P}<0.05$ was considered as statistically significant

SV: Stroke Volume; LVET: Left Ventricular Ejection Time; CO: Cardiac Output; TPR: Total Peripheral Resistance; BRS: Baroreflex Sensitivity

Table 4: Pearson correlation of TP with various parameters in control group $(\mathrm{n}=30)$ and study group $(\mathrm{n}=27)$.

\begin{tabular}{ccccc}
\hline Parameters & \multicolumn{2}{c}{ Control Group } & \multicolumn{2}{c}{ Study Group } \\
\hline & $\boldsymbol{r}$ & $\mathrm{P}$ & $\boldsymbol{r}$ & $\mathrm{P}$ \\
\hline BHR & 0.130 & 0.180 & -0.280 & 0.021 \\
SBP & 0.095 & 0.411 & -0.256 & 0.056 \\
DBP & 0.160 & 0.119 & -0.268 & 0.047 \\
RPP & 0.108 & 0.287 & -0.162 & 0.143 \\
TPR & 0.205 & 0.087 & -0.260 & 0.055 \\
BRS & 0.215 & 0.062 & 0.247 & 0.047 \\
\hline
\end{tabular}

BHR: basal heart rate; SBP: systolic blood pressure; DBP: diastolic blood pressure; RPP: rate pressure product; TPR: total peripheral resistance; BRS: baroreflex sensitivity.

P Value $<0.05$ was considered significant.

However, Baroreflex sensitivity (BRS) was reduced significantly in the study group compared to the control group.

Table 4 shows the correlation between total power (TP) and other parameters of the control and study groups. In the control group, the TP did not reveal a significant correlation with the other study parameters. However, a significant negative correlation was observed in the study group between TP, BHR and DBP, and a significant positive correlation between TP and BRS.

\section{DISCUSSION}

In the present study, twenty-seven patients diagnosed with generalised tonic-clonic seizures and on treatment for the same for the past one year were included in the study group. Age and gender-matched subjects from the normal population were included in the control group. The prevalence of cardiovascular risk in people living with epilepsy (PWE) was compared with the normal population in the present study.

There was no significant difference in age and body mass index (BMI) of the study and control group, as these parameters are known to affect the autonomic functions and CV risk parameters. Hence, it may be stated that the observed changes in the outcome variables may not be due to the effect of age and BMI.

Basal heart rate (BHR) denotes the function of the cardiac vagal tone. An increase in BHR is a risk factor for CV disease. ${ }^{[9,10]}$ BHR was significantly high in PWE compared to the normal population reflecting a decreased vagal tone. Systolic blood pressure (SBP) reflects sympathetic activity. There was a significant rise in SBP in PWE, reflecting the increased sympathetic activity. Similarly, diastolic blood pressure (DBP) denotes the basal sympathetic vasoconstrictor tone. Significantly -raised DBP was observed in PWE, reflecting the increased sympathetic vasoconstrictor tone. Rate pressure product (RPP), a measure of myocardial workload and oxygen consumption ${ }^{[5]}$ was significantly high in PWE, indicating increased myocardial work stress. The above findings reflect an imbalance of sympathetic and parasympathetic systems i.e., autonomic dysfunction in PWE compared to the normal population. This was further supported by heart rate variability (HRV) parameters which reflect the cardiac sympathovagal status.

With respect to frequency domain indices of HRV, total power (TP) was significantly low in PWE. TP reflects the overall strength of cardiac vagal modulation. Time domain indices of HRV represent vagal drive to the heart. ${ }^{[4]}$ Among time domain indices, RMSSD is considered as an important marker of cardiac beat to beat parasympathetic drive. ${ }^{[4,11]}$ There was a significant decrease in RMSSD in the study group compared to the control group, reflecting the decreased parasympathetic drive. ${ }^{[4]}$

In this study, the BP parameters were recorded by BPV analysis system using Finapres. BRS has been established as a standard non-invasive marker of $\mathrm{CV}$ risk, and BRS indicates an integrated activity of the sympathetic and parasympathetic systems. BRS has been reported to be an index of sympathovagal imbalance in various $\mathrm{CV}$ disease states. ${ }^{[6]} \mathrm{BRS}$ was found to be significantly low in the study group compared to the control group, reflecting the sympathovagal imbalance and an increased $\mathrm{CV}$ risk in these individuals. Total peripheral resistance is considered a marker of resting sympathetic vasoconstrictor discharge. ${ }^{[12]}$ In this study, TPR increased in the study group, indicating an increase in the sympathetic vasoconstrictor tone in them compared to control group. These findings were further substantiated by the correlation test results, which revealed a significant negative correlation between TP, BHR and $\mathrm{DBP}$, and a significant positive correlation between TP and BRS.

\section{CONCLUSION}

In conclusion, decreased TP, BRS and increased BHR, SBP, DBP and RPP in the study group indicates cardiac sympathovagal imbalance and an increased risk of cardiovascular events in PWE compared to the normal population.

\section{Limitations of the Study}

The study has the following limitations: 1 . Considering the age group of the study participants, the study results cannot be generalized to the entire population. 2. Body composition and body fat analysis, which could indicate and contribute to autonomic imbalance and CV risk were not assessed in the participants.

\section{ACKNOWLEDGEMENT}

Jawaharlal Institute of Postgraduate Medical Education and Research, Puducherry, for providing financial assistance in the form of intramural MD research grant.

\section{CONFLICT OF INTEREST}

The authors declare no conflict of interest.

\section{ABBREVIATIONS}

PWE: People living with epilepsy; CV: Cardiovascular Risk; BPV: Blood Pressure Variability; HRV: Heart Rate Variability; HR: Heart Rate; SBP: Systolic Blood Pressure; DBP: Diastolic Blood Pressure; TP: Total 
Power; BRS: Baroreflex sensitivity; TP: Total Power; BHR: Basal Heart Rate; ILAE: International League Against Epilepsy; RPP: Rate Pressure Product; CO: Cardiac output.

\section{REFERENCES}

1. Daniel H. Lowstein Harrison's manual of Medicine. 19 ${ }^{\text {th }}$ ed. New York: McGrawHill Education; p. 2542.

2. Thijs RD, Surges R, O'Brien TJ, Sander JW. Epilepsy in adults. Lancet 2019;393(10172):689-701. doi: 10.1016/S0140-6736(18)32596-0, PMID 30686584.

3. Myers KA, Bello-Espinosa LE, Symonds JD, Zuberi SM, Clegg R, Sadleir LG, et al. Heart rate variability in epilepsy: A potential biomarker of sudden unexpected death in epilepsy risk. Epilepsia. 2018;59(7):1372-80. doi: 10.1111/epi.14438, PMID 29873813.

4. Heart rate variability: Standards of measurement, physiological interpretation and clinical use. Task Force of the European Society of Cardiology and the North American Society of Pacing and Electrophysiology. Circulation. 1996;93(5):1043-65. doi: 10.1161/01.CIR.93.5.1043, PMID 8598068.

5. White WB. Heart rate and the rate-pressure product as determinants of cardiovascular risk in patients with hypertension. Am J Hypertens. 1999;12(2 Pt 2): 50S-5S. doi: 10.1016/s0895-7061(98)00280-5, PMID 10090295.
6. La Rovere MT, Pinna GD, Maestri R, Sleight P. Clinical value of baroreflex sensitivity. Neth Heart J. 2013;21(2):61-3. doi: 10.1007/s12471-012-0349-8, PMID 23184601.

7. Imholz BPM, Wieling W, Van Montfrans GA, Wesseling KH. Fifteen years experience with finger arterial pressure monitoring: Assessment of the technology. Cardiovasc Res. 1998:38(3):605-16. doi: 10.1016/s00086363(98)00067-4, PMID 9747429.

8. Pal GK, Adithan C, Amudharaj D, Dutta TK, Pal P, Nandan PG, et al. Assessment of sympathovagal imbalance by spectral analysis of heart rate variability in prehypertensive and hypertensive patients in Indian population. Clin Exp Hypertens. 2011;33(7):478-83. doi: 10.3109/10641963.2010.549275, PMID 21978027.

9. Palatini P. Heart rate and the cardiometabolic risk. Curr Hypertens Rep 2013;15(3):253-9. doi: 10.1007/s11906-013-0342-7, PMID 23645136.

10. Jensen MT, Suadicani P, Hein HO, Gyntelberg F. Elevated resting heart rate, physical fitness and all-cause mortality: A 16-year follow-up in the Copenhagen Male Study. Heart. 2013;99(12):882-7. doi: 10.1136/heartjnl-2012-303375, PMID 23595657.

11. Malliani A. Heart rate variability: From bench to bedside. Eur J Intern Med. 2005;16(1):12-20. doi: 10.1016/j.ejim.2004.06.016, PMID 15733815.

12. Pal GK. Regulation of blood pressure. In: Comprehensive textbook of medical physiology. New Delhi: Jaypee publications; 2017b;2:822-48.

Cite this article: Sravanthi KL, Pal GK, Nair PP, Rajalakshmi R, Nanda N. Assessment of Cardiovascular Risk in People Living with Epilepsy. Int J Clin Exp Physiol. 2021;8(3):118-21. 